\title{
Language in Academic Writing: Features and Topical Issues
}

\author{
Ifeyinwa Awagu \\ Institute of Humanities, Pan-Atlantic University, Ibeju-Lekki, Lagos-Nigeria
}

\begin{abstract}
.
The quality of language of a scholarly paper determines its acceptability for academic publication. Different academic publications have house styles that include language, referencing and citation. Whether books, editorials or journals, there are distinguished styles of expressions, sentence construction, lexicon and mode of writing or reporting within the academic domain. Given that authors are expected to communicate the organized thoughts and research findings with accuracy and sensitivity, challenges abound in conformity to house style or grammar. Sometimes, the authors encounter the interference of structural construction or the interference of the mother tongue for a non-native speaker, writing in a second language. Through a review of the literature and guidelines laid by some academic house styles like APA 6th Edition, this paper examines the specific features of language that an author writing in any language for publication need to consider for acceptability. The paper posits that scholarly research is a specialty, thus the language is within the unique set of rules governing academic writing. The nature of some scholarly research disposes the report to an exclusive audience which might demand language conceptualized within the boundaries of the discipline. Language barrier between thoughts and written words constitute hindrance for non-native speakers. Academic writing needs special tutoring in the language both for native and non-native speakers. Academic brokerage should be encouraged.
\end{abstract}

Keywords: authorial-presence, clarity, objectivity; scholarly-language 


\section{2nd International Conference on Research in SOCIAL SCIENCES and HUMANITIES}

\section{Introduction}

Scholarly or academic research is often characterized by some distinguished style of expression that defines its intellectual boundaries. These expressions accord them specific language and mode of writing within the discipline or domain of expertise. The language is crafted to convey agreed features and meaning in line with the characteristics of scholarly research, as planned and focused, structured, evidenced, and formal in tone (Hartley, 2008; University of Leeds). Language is a tool employed to convey information, ideas and thoughts, all geared towards facilitating human communication. Specific instances of language use are bound to the material world of the users, thus, driving language to be context specific (Victoria Institutions, 2011).

While the nature of some scholarly research disposes the report to an exclusive audience, others are accessed by diverse audiences. The fact that it is a scholarly research keeps the language within the unique set of rules governing academic writing. It is prime in the evaluative criteria for publishable academic writing. The efforts of scholars to establish the gulf between the language of basic communication and that of academic writing generated common features in the language of scholarly research.

Authors write and rewrite academic articles for publication, but sometimes suffer rejection on account of non-adherence to the language of the academic writing. Some authors identify challenges in conformity to house style and grammar, or authors' choice of effective words to communicate the organized thoughts with accuracy and sensitivity (Lang, 2017). Sometimes, the authors encounter the interference of structural construction or the interference of the mother tongue for a non-native speaker, writing in a second language.

Through a review of the literature and guidelines laid by some academic house styles like APA 6th Edition, this paper examines the specific features of language that an author writing in any language for publication need to consider for acceptability. The paper posits that scholarly research is a specialty, thus the language is within the unique set of rules governing academic writing. The nature of some scholarly research disposes the report to an exclusive audience which might demand language conceptualized within the boundaries of the discipline. Language barrier between thoughts and written words constitute hindrance for non-native speakers. Academic writing needs special tutoring in the language both for native and non-native speakers. Academic brokerage should be encouraged.

\section{Scholarly Research and Language of Scholarly Research or Academic Language}

Scholarly research also called academic research, represents properly or scientifically conducted research or experiment, with originality, by one or more scholars. The research is intended to be made available to the scholarly world through publication in books or scholarly journals. They are subjected to reviews and comments by the academics or experts in the field, in order to qualify for publication. The publications are discipline specific or multidisciplinary; example, Journal of Cross-Cultural Psychology is published by Cultural 


\section{2nd International Conference on Research in SOCIAL SCIENCES and HUMANITIES}

and Social Psychologists but accepts articles in related fields like anthropology and other cultural and behavioral studies.

The review and comment earn the scholarly journals the term, peer-reviewed or refereed journals. They often have prescribed format for the researched articles: the title, the abstract, and the main text. The title states the subject matter while the abstract is the summary. The main text is the content and often adds graphs and charts. Other scholarly writings relevant to the research are cited and credited in accordance with the house style. For example, APA 6th Form uses Reference List in place of Bibliography. The researcher's affiliations, such as the universities, research institutions, etc., are also listed according to the house style or usually at the bottom of the first page or at the end of the article.

The language of scholarly research or academic language is not a specific language of communication with unified meaning, such as English or French. Rather, a style or mode of expression, a specialist language adopted within an educationally relevant and methodological framework; and for an intended audience. The language includes all the textual materials employed by the writer to interact with the reader. Gottlieb \& Ernst-Slavit (2013) referred to it as a complex concept. The fact that the purpose, audience, and context varies, results in clear differences in terms of language use, in the selection of words, formality, sentence construction, and discourse patterns.

Snow and Uccelli (2009), affirming Scarcella (2003) specifically described academic English as "a variety or register of English used in professional books and characterized by the linguistic features associated with academic disciplines." This was validated by Gottlieb \& Ernst-Slavitn (2013) that described academic language or academic English as a register, or an assortment of a language used for a specific purpose and audience in a particular context. It is typified by the specific linguistic features associated with the academic disciplines, including discourse features, grammatical constructions, and vocabulary across different language domains, modalities and content areas. It is multidimensional and dynamic in nature; each variance evolves as the discipline evolves, but they all share certain core features (Halliday, 1993 as cited by Gottlieb \& Ernst-Slavitn, 2013).

The peculiar technicalities of the specific discipline, the academic community, as well as clear and formal communication are observed. Though the former might present some difficulty in understanding for some readers, the fact that the research is meant for a target academic landscape cannot be lost in popular readability. The pendulum of style of language dangles between expertise and clarity. Proficiency in the use of academic language demands the ability to use content-specific vocabulary, appropriate or specialized grammatical structures, and discourse in disseminating the research information.

Hartley (2008) identified a clear focus on the research problem under investigation, a formal tone, precise word choice, and use of the third-person rather than first-person perspective, as the characteristics of academic writing.

\section{Distinctive Features of Scholarly Language}

The scholarly research has a communicative purpose which constitutes the rationale that determines the schematic structure of discourse and constrains the rhetorical choice. The language is established and accepted by the culture of a specific discourse community whose members share the broad communicative purposes (Wu, \& Zhu, 2014). Scholarly language, 


\section{2nd International Conference on Research in SOCIAL SCIENCES and HUMANITIES}

though subject to specific genres, is explicit, formal and factual as well as objective and analytical in nature. It is designed to be very precise, concise, courteous and authoritative. The features of scholarly language can be discussed along the following titles: objectivity, clarity, analytical and authorial presence.

\section{i) Objectivity}

Objectivity is concerned about facts based on the research and not opinion and biases; nor facts influenced by opinion and biases. The writer assumes an authoritative stance, stating strengths of the argument confidently. The language presents arguments and not value judgements. It is neither meant to be confrontational nor dismissive, but sometimes, these are necessitated by the academic environment. The perspectives contrary to that of the writer are given the appropriate narrative tone that conveys fairness and objectivity. The argument needs to be described accurately without biased expressions. The tone is also a factor in establishing the writer's position. It has to be convincing.

Bias as against objectivity in scientific writing is contained in evaluative statements that are overt or implied. The writer has to avoid the use of words or expressions that are judgmental of race, gender, sexual orientation, or any identifiable group. Bias can also be by way of assumptions or constructions or use of sensitive labels to describe groups.

Another angle in discussing objectivity as a feature of scholarly language is the place of a convincing language. Much as scholarly research is largely a matter of documentation, rigorous testing, and other objective controls, a formal convincing language is essential. Some critics argue that no matter how "objective" your facts, statistics, results of experiments, or quotations from respected sources, the argument is still by its very nature "subjective" (Kendra, 2013). Given that it is a hypothesis that is investigated and proven, the language is such that the readers will be convinced.

The language of scholarly research demands objectivity in expression. The author needs to demonstrate a clear focus on the research problem under investigation. Objectivity can be reflected in the writers' approaches to the development of the topic, the written discourse paradigm, and use of syntactic and referential markers (Hinkel, 1999). Academic discourse communities have the prescribed level of normativity for rhetorical and syntactic features that express objectivity. Atkinson (1991) contends that the superordinate notion of "scientific objectivity" is partly conventional in origin. In some established territories, the pronouns "we" and "us" are appropriate alternatives for "I" and "me". Dialectical and cultural variances are identified in objectivity devices and markers in rhetorical conventions acceptable in English speaking societies. Thus, common linguistic medium does not mean that discourse strategies - rhetorical devices, syntactic and referential markers denoting objectivity - are shared (Hinkel, 1999).

The choice of words is such that communicate the context of the writing the best way. Use of vocabularies, with meanings that are very specialized to the discipline, are important. They enable accurate understanding of concepts within the text. Frugality with words makes the article more readable, so the technical terminologies should be concise. An appropriate level of specificity in the choice of words helps to eliminate bias.

From the title of the scholarly research, through the abstract, to the main text, informative expressions testify to objectivity. Declarative and interrogative titles are less objective 


\section{2nd International Conference on Research in SOCIAL SCIENCES and HUMANITIES}

because either they are incomplete or call attention only to a part of the research (Lang, 2017). The type of research article determines the language of the abstract. Descriptive expressions are commonly used in review abstracts.

\section{ii) Clarity}

Ideas are stated as concisely as possible. Clear writings avoid vague expressions, sweeping statements and repetitions. It does not employ words open to the possibility of the reader misinterpreting the design and purposes of the study. The concrete words and terminologies that convey specific meanings are used, while those that can evolve nuanced meaning within the discipline are avoided. Much as appropriate use of specialist language and a formal style of expression are allowed, excess use of complex or obscure words; and complicated sentence constructions give the impression that the article is more about style than substance (USC Libraries, n.d). Consistency in use of identical conceptual language in the text creates clarity.

A chosen tense is more appropriate, but the results of the findings are usually expressed in the past tense. The APA 6th edition recommends the use of the past tense when reporting one's result or discussing another researcher's work; but present tense in discussing the implications of the result and the conclusion. Examples, "Tsaaior (2016) indicated that;" "This study demonstrated that;" "The import of the result is that."

It is better to write in positive terms. Example, "the findings revealed that" is clearer than "the findings did not reveal." Short sentences are more effective. Smooth run of expression is achieved by use of simple sentences. The structure of the text aids explicit presentation of ideas to the extent that the reader follows. This demands adherence to the grammatical structure of the given language. For example, the common pitfalls in the English language are the use of fragmented sentences, dangling modifier, punctuation, etc. Nominalizations of verbs are used only when appropriate.

The importance of grammatical correctness to clarity is validated in Zare et al (2016) which investigated scholarly peer-review comment for research publication in English Language. The study observed that regardless of discipline, "lexis and syntax" attracted considerable comment compared to "discourse and rhetoric" under scholarly language employed in the manuscripts submitted for peer review. The Iranian non-native speakers of English language researchers' submissions came as an example of the clear indication that the referees of international high profile journals find syntactic and lexical use of English much more problematic than text structure. Specifically, "lack of clarity", ranked the most frequently commented themes in the manuscripts among others. Lexis and syntax feature as important aspects of clarity and precision, which are the building blocks of academic writing. It added that academic writing training, hence, should address such issues as balance in the use of active and passive voice, consistency in the use of tense, the natural sequence of the parts of speech, lexical choice of scientific terminology, clarity, and brevity.

However, some disciplines seem to weigh clarity in discourse and rhetoric against clarity in lexis and syntax. Zare et al (2016) pointed out that the humanities researchers stressed the superiority of the transitions or link from one section of the research article to another. The knowledge of the moves of the different sections of an article is more fundamental in the academic writing training of this group of researchers. They may need to focus on how to manage the transitions of the different sections of an article to make for clarity and brevity, more than the emphasis on rhetorical devices. 


\section{2nd International Conference on Research in SOCIAL SCIENCES and HUMANITIES}

In conclusion, clarity in writing reflects on clarity in thought. Academic research is far more than mere fact recording. The language is a key to transmitting knowledge and rendering an impact on the field. Without clarity and proper use of language, readers will not grasp the full message or impact of the article. Even with cutting edge findings, unconventional language quality, including errors in grammar, spelling or language usage, could render the article unacceptable for publication (Griffies, Perrie and Hull, 2013)

\section{iii) Analytical}

The subject matter of scholarly research resides in empirical perspectives of knowledge. An appreciation of the intricacy of this subject matter is demonstrated through critical analysis. Use of analytical language aids a critical presentation of both the perspectives in review and the new perspectives. This language approach enables the writer to dissect all sides of the subject matter by way of contrast, comparison, explanation, validation and such like. Hartley (2008) opines that it also means that you explain, give reasons, draw conclusions, make suggestions and recommendations and support this with evidence.

The language style of scholarly research is primarily argumentative. Arguments are presented to support claims, therefore, have to present a thorough and consistent reasoning. The use of clear topic sentences in well-structured paragraphs enable the reader to follow the line of argument without difficulty. Though the narrative links between sentences and paragraphs are needed to enable the reader to follow the argument, as is common in other forms of writing, the structure is formal, cohesive and logical. The various parts must be connected to form a unified whole.

Various linguistic styles are employed to engage the reader while sustaining the argument. Swales, Ahmad, Chang, Chavez, Dressen and Seymour (1998) examined the use of imperatives in scholarly writing. The study scanned for imperatives used in both main text and notes, and instances were collated and analysed. The findings revealed that main-text imperatives tend to congregate in sections where the principal argumentation occurs, but are very unevenly distributed across fields. As such, the authors use them for various strategic purposes such as engaging the reader, achieving text economy, or manifesting personal style.

\section{iv) Authorial Presence}

Authorial presence is the way the writer reveals his identity in his relationship with the readers and the academic community within the research article. There are diverse conventions of different disciplines and academic writing cultures, on how and the extent to which writers can explicitly visible in the discourse. These variations made the mode of language employed in establishing authorial presence seem highly problematic for writers and students alike. The choices range from the self-effacing task which involves authors eradicating themselves from their texts through third person narratives; to self-citation with exclusive first pronouns (Hyland, 2001).

The scholarly language of some academic writing cultures demands a presentation of the research in the third person narrative and not first person. The authorial presence is not usually direct in some traditions. It is often manifested in the tone, use of personal pronouns and directives in the writing. The use of I, we, me, us, you, etc., infuse subjectivity in the research and rob it of empirical evidence. This is not generalized to all traditions and genres. The personal pronoun "we" is acceptable in some traditions, whereas some traditions indicate 


\section{2nd International Conference on Research in SOCIAL SCIENCES and HUMANITIES}

the author's voice in the phrase "the researcher" or "researcher" (Shehzad and Sohail, 2012). The self-mention is in the form of detached self. Other forms are the individual self and the collective self.

The author assumes an objective stance in presenting the body of knowledge and academic debate related to the subject matter. The language is such that threads a well-documented, coherent, and logically structured piece of writing without direct visibility of the author. This is particularly important when proposing solutions to problems or recommended courses of action (USC Libraries, n.d). Conventional language of citation in the body of the work is employed to show the source of the voice, and all sources are properly cited.

The scientific writing is identified by its stand on the issues of truth, thought and language. There is need to present the new result coherently within the existing body of knowledge. This stance makes the scientist replaceable in that the experiment or research could be replicated by any other person. The authorial invisibility emphasizes this philosophy. However, some traditions allow the author's voice to come through the use of the first person pronouns in the introduction, conclusion, proposing new approach, encouraging a new direction, or suggesting new design; but not in the experimental results (Mark, 2018).

Wu \& Zhu (2014) explains that following the social semiotic view that language performs social functions, the research article is not taken as purely objective reporting of an independent and external reality. Rather, it should be seen as a narration of investigation, a process of reconstructing research, and a channel for distributing and advancing academic knowledge. As such, it involves two levels of communication: the convincing style of the research report and the appropriate presentation of authorial self to readers to enhance his reputation. They concluded that research articles not only convey disciplinary content, but also establish the desired authorial presence, through the linguistic choices. These are embedded in the scholarly language of the genre.

The combined effect of the language of authorial presence is validated in an earlier research by Hyland (2001) emphasizing that the choices which express the writer's presence not only affect the ideational meaning that writers convey, but also influence the impression they make on their readers. The import of Hyland (2001) and $\mathrm{Wu} \& \mathrm{Zhu}$ (2014) is that the choice of scholarly language of the research article establishing authorial presence is guided by the purpose of the self-mention. Self-mention is deemed as a powerful rhetorical strategy of emphasizing a writer's contribution (Hyland, 2001)

A cross-cultural approach to the analysis of the linguistic elements of authorial references was adopted in Molino (2010). Specifically, the use of the first person subject pronouns and passive voices in research articles in English and Italian were analyzed to determine whether personal and impersonal authorial references as realized by these features are susceptible to variation across academic writing cultures. The results indicate variations that were ascribed to the adoption of differing interpersonal strategies, subjectivity or objectivity, within the two academic discourse communities.

The existence of different scholarly traditions triggers a line of inquiry. The extent to which culture specific practices in the linguistic manifestations of the writer's persona ( eg. use of personal pronouns as self-mention devise, use of attitudinal lexis) establish credibility, highlight research outcomes and ultimately seek the acceptance of the community of experts 


\section{2nd International Conference on Research in SOCIAL SCIENCES and HUMANITIES}

(Pérez-Llantada, 2009) This is evident in the pressure faced by non-native speakers of language publishing research in international journals.

Given the presumed dominance of English Language in international publications, PérezLlantada (2009) assessed native and non-native English speakers along the line of choice of linguistics and rhetorical devices in the scholarly research written in English. The study complemented interview data with textual analysis to quantify a sample of linguistic features and interpret them as rhetorical devices that writers use to construct new knowledge, evaluate research processes and highlight research outcomes. The textual analysis revealed several intercultural differences as regards the degree of authorial visibility/ invisibility in the texts. It advocated the maintenance of cultural diversity in international publications. Such scholars may retain some culture-specific traits, while adapting to the rhetorical conventions for writing papers in the second language. Using Spanish as an example, it highlighted that the tradition keeps authorial visibility to a minimum.

Directives are best framed as evidence-based recommendations, not as instructions that demand the readers to do one thing or the other. Conversational or informal tones such as use of slangs and idioms are not permissible. Expressions specific to regional dialects expose the writing to variances in interpretation.

\section{Topical issues on features of scholarly language.}

The linguistic variances, in terms of academic research, across different cultural and national traditions are issues in the use of scholarly language. Scholars sometimes write in their native language for an international audience. While such writers may compose an academic text that fully corresponds to the local/ national norms and fits the expected lexicogrammatical and rhetorical patterns in the writer's native academic culture, the same situation might not correspond with the translated text meant for an international publication (Chovanec, 2012). A discourse of the features of the language of scholarly research in a crosscultural context lends itself to practical dimensions in socio-linguistic, ethnographic, translationlogical and pedagogic orientation. For example, a translation of a scholarly research from English to German faces the dilemma of what approach to take with respect to the translation, particularly in terms of its textual and rhetorical structure.

While investigating the role of peer reviewers in accepting or rejecting manuscripts with off-network sources, Zare, Mahmoudi-Gahrouei, Ketabi \& Keivanloo-Shahrestanaki (2016), cited Belcher (2007) that examined the submission history of an applied linguistics journal. The findings revealed that language use and style are the most frequently highlighted areas of the reviewed articles that place them below the required standard for publication.

There is a challenge in an attempt to make it correspond more closely to the conventions of the target academic culture in order to increase the article's chances of publication. The options are either surface-level adjustments or other significant textual reorganization. This might amount to essentially re-writing the text, possibly ending in a clash of the different scholarly language styles and academic experiences. Existing studies recommend a pedagogical approach of instruction and guidance in the styles and genres of written academic discourse in the second language to acquire skills that are proving increasingly indispensable in the modern marketplace. This can develop their sensitivity to different styles, cultivate their ability to switch between diverse styles, and enhance their skills in formulating spoken and 


\section{2nd International Conference on Research in SOCIAL SCIENCES and HUMANITIES}

written messages in ways that are maximally effective, and take into account the context of the target culture/situation/audience (Chavenec, 2012)

Mediation of academic literacy in scholarly research is another prevalent issue. It refers to the various ways that literary brokers help writers interact with the written text, within and outside contractual relationships (Lillis \& Curry, 2006)). Though little research has explicitly focused on mediation in relation to scholarly research, the notion of mediation is evident in Burrough-Boenisch (2003) as cited by Lillis \& Curry (2006). The study, while looking at text interventions, explores hypothetically the activities of those the authors referred to as "shapers" of texts written by Dutch-speaking scientists. These are language professionals journal reviewers and editors - as well as members of the author's discourse community, whose extensive intervention brings the research to the standard of publication in an international journal. This is mediation in writing scholarly research informed by the discourse community.

Lillis \& Curry (2006) examined the phenomenon of literacy brokering in academic text production, which, although a familiar practice to many publishing academics, has been the object of little systematic empirical research. The study specifically investigated the experiences of multilingual scholars who live and work in periphery contexts of the nonAnglophone center. Using a text-ethnographic approach, it revealed the extent and nature of literacy brokering in broad terms and explored in detail the trajectories of three texts as they move from local peripheral scholarly research contexts (national publication within their locality) toward publication in the center (English-medium international journal). It established that there are different orientations in mediation according to the need for the mediation: language or content focused. An important finding is that though scholars tend to frame brokers' interventions in terms of language or discourse, the brokers tend to orient to content.

Apart from the key finding in Lillis \& Curry (2006), it observed that the rhetorical changes instigated by brokers can be understood as Western Anglo academic literacy practices, where specific notions of textual unity and, conversely, digressions are privileged. Such rhetorical practices are dynamic even within center disciplinary contexts. There is an example of brokers from two different English-medium international journals with different approaches to the rhetorical treatment and function of experiments.

The relevance of the findings to this discussion lies in the intricacies of standard of language in scholarly research among multilingual scholars in relation to publication in peer reviewed international journals published in dominant international languages such as English. The precise nature of the activities both groups of scholars: the non-native speaker, writing in the language of the native speaker who is the broker in the mediation process lends the mediation to unequal power play. It makes the terrain of acceptability of the scholarly research of such scholars, for international publication, more limited and complex. Mudrak, (2013) opined that international authors sometimes perceive biases based on language skills, country of origin, or other factors. The study found that reviewers and editors frequently comment on grammar and diction in manuscripts from second-language authors. It cited an example of a study of medical manuscripts written by Italian researchers where it was found that $44 \%$ of all reviewer comments dealt with language issues. 


\section{2nd International Conference on Research in SOCIAL SCIENCES and HUMANITIES}

Interestingly, Mudrak, (2013) observed that helpful comments on the language in a manuscript are completely appropriate for a review, but mentioning a large number of language issues can make the author feel defensive and may even create concern that the manuscript is being judged more harshly than it would have been if the manuscript had been written by a native English speaker. By way of recommendation, the study added that to combat the possibility of perceived bias, author instructions should include a statement stressing the need for a certain threshold of English language quality. This provides reviewers the opportunity to properly assess their content, and by explaining the rationale behind any comments on the language in a manuscript, such comments can be taken constructively and not in a pejorative manner.

Despite the ongoing definitions, a conceptualization of academic language within an agreed logical framework that could guide educationally relevant research is a challenge. The focus of such framework ought to supersede the linguistic features for a practice that would generate a better directly useful information, like analysis of the content standards, discourse, etc. (Snow and Uccelli, 2009).

A systematic and critical approach to scholarly language ought to draw on the factors such as the discipline, intellectual traditions, and genre and writing styles. This would demonstrate recognition of the complexities of academic cultures and communities.

\section{Conclusion and Recommendation}

Editors give priority to manuscripts that are clearly written. No matter the quality of an academic research, it is the language of the report that communicates and authenticates the findings. The language of scholarly research is permeated with specific characteristics that accommodate the peculiar technicalities of the specific discipline, the academic community, as well as clear and formal communication. These are evident in the rhetorical and syntactic features and house style of academic discourse communities. Given that the objective is to present a clear communication of the scholarly research, the rubrics have to be adhered to. These include observance of the grammatical structure and rules of the specified language.

By way of appropriate use of scholarly language, publishing research in one's native language is difficult enough, but second-language authors face additional challenges (Mudral, 2013). The language barrier generates certain levels of complexities for both the scholarauthor and the scholar-reviewer. These can be scaled through some measures, such as providing reviewers' information about writing in clear language to improve the exchange between international authors and reviewers.

Lillis and Scott (2007) expressed concern to explore the complexities involved in academic communication, and particularly writing, in the face of what are often powerful but restricted and deficit official discourse or language. They employed the term "academic literacy" as a dynamic phenomenon embedded in - rather than separate from - research activity; thus, advanced a common level of understanding for scholars in scholarly research and publication that will emphasize a shared communicative purpose.

Finally, the language of scholarly writing is that which expresses simply, specifically, formally and convincingly, that which it is meant to express. The readability does not need special skills, but has to go beyond the language of the ordinary. 


\section{2nd International Conference on Research in SOCIAL SCIENCES and HUMANITIES}

A discourse of the features of the language of scholarly research in a cross- cultural context lends itself to practical dimensions in socio-linguistic, ethnographic, translation logical and pedagogic orientation

It would be a good consideration for academic communities not represented in high impact factor journals to grow journals with high impact factor within national or cultural boundaries

\section{Acknowledgment (TNR 12pt. bold)}

This paper is an output of an assignment I wrote for my PhD Course 


\section{2nd International Conference on Research in SOCIAL SCIENCES and HUMANITIES}

\section{References}

Chovanec, J. (2012). Written academic discourse in English: From local traditions to global outreach. Brno Studies in English Volume 38, No. 2

Gottlieb, M. \& Ernst-Slavit, G. (2013). Academic Language: A Centerpiece for Academic Success in English Language Arts PP 1-8. Retrieved from https://us.sagepub.com 2014

Griffies, S. M., Perrie, W. A. \& Hull, G. (2013). Elements of style for writing scientific journal articles. Retrieved from Elsevier. https://www.publishingcampus.elsevier.com

Hartley, J. (2008) Academic Writing and Publishing: A Practical Guide. New York: Routledge

Hinkel, E. (1999) Objectivity and credibility in L1 and L2 academic writing in Hinkel, E. (Ed) Culture in second language teaching and learning. Cambridge: Cambridge University Press p 3-10

Hyland, K. (2001) Humble servants of the discipline? Self-mention in research articles. English for Specific Purposes. Volume 20(3) pp 207-226. Retrieved from Science Direct. http://www.sciencedirect.com

Kendra, G. (2013). Graduste writing resource. Retrievd from The University of Arizona https://www.gwr.arizona.edu/balancing1.htm

Lang, T. A. (2017). Writing a better research article in Journal of Public Health and Emergency. Retrieved from JPHE. http://jphe.amegroups.com/article/view/4265

Lillis, T. \& Curry, M. J., (2006) Professional academic writing by multilingual scholars: interactions with literacy brokers in the production of English-Medium texts. Written Communication 23 (3). Retrieved from Sage Publications. http://wcx.sagepub.com/

Lillis, T. \& Scott, M. (2007) Defining academic literacies research: issues of epistemology, ideology and strategy. Journal of Applied Linguistics. Vol. 4 Issue 1, pp5-32. Retrieved from http://web.a.ebscohost.com 